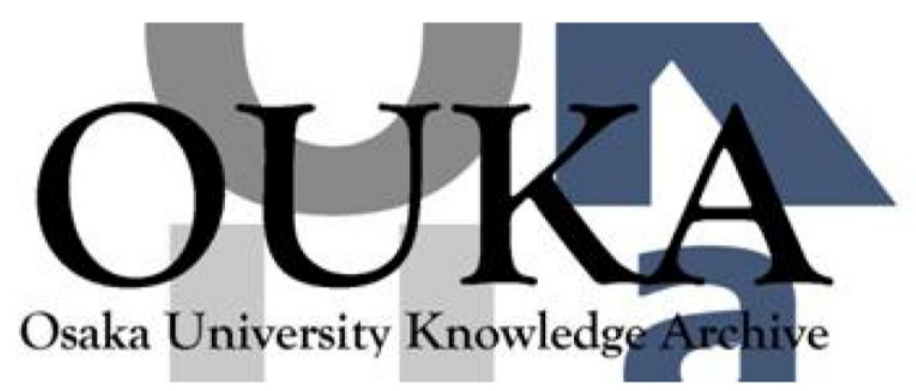

\begin{tabular}{|c|c|}
\hline Title & $\begin{array}{l}\text { Electronic band structure and magnetism of } \\
\text { Fe16N2 calculated by the FLAPW method }\end{array}$ \\
\hline Author(s) & $\begin{array}{l}\text { Tanaka, Hirofumi; Harima, Hisatomo; Yamamoto, } \\
\text { Tetsuya et al. }\end{array}$ \\
\hline Citation & Physical Review B. 62(22) p. 15042-p. 15046 \\
\hline Issue Date & $2000-12$ \\
\hline oaire:version & VoR \\
\hline URL & https://hdl. handle. net/11094/3044 \\
\hline rights & $\begin{array}{l}\text { Tanaka, Hirofumi, Harima, Hisatomo, Yamamoto, } \\
\text { Tetsuya, Katayama-Yoshida, Hiroshi, Nakata, } \\
\text { Yoshiyuki, Hirotsu, Yoshihiko, Physical Review } \\
\text { B, 62, 22, 15042-15046, 2000-12. "Copyright } \\
2000 \text { by the American Physical Society." }\end{array}$ \\
\hline Note & \\
\hline
\end{tabular}

Osaka University Knowledge Archive : OUKA

https://ir. Library. osaka-u. ac. jp/

0saka University 


\title{
Electronic band structure and magnetism of $\mathrm{Fe}_{16} \mathrm{~N}_{2}$ calculated by the FLAPW method
}

\author{
Hirofumi Tanaka, ${ }^{*}$ Hisatomo Harima, Tetsuya Yamamoto, ${ }^{\dagger}$ Hiroshi Katayama-Yoshida, Yoshiyuki Nakata ${ }^{\ddagger}$ \\ and Yoshihiko Hirotsu \\ The Institute of Scientific and Industrial Research, Osaka University, 8-1 Mihogaoka, Ibaraki, Osaka 567-0047, Japan
}

(Received 5 April 1999; revised manuscript received 7 September 2000)

\begin{abstract}
Electronic band structure calculations based on the full-potential linear augmented plane-wave method have been performed for $\mathrm{Fe}_{16} \mathrm{~N}_{2}$. The calculations are performed with the crystal parameters recently refined [H. Tanaka et al., Acta Mater. 45, 1401 (1997)] in addition to the previous reported structure [K. H. Jack, Proc. R. Soc. London, Ser. A 208, 200 (1951)]. Jack's model ( $J$ model) led to the model of Tanaka et al. ( $T$ model), where Fe atoms at the $8 h$ site shift to $\mathrm{N}$ atoms along the [110] direction. The calculated average moment per Fe atom for the $T$ model is slightly smaller than that for the $J$ model. We cannot theoretically expect a large magnetic moment based on any $\mathrm{Fe}_{16} \mathrm{~N}_{2}$ structure. The presence of another material that has a large magnetic moment must be considered to explain the large magnetic moment of the $\mathrm{Fe}-\mathrm{N}$ system.
\end{abstract}

\section{INTRODUCTION}

In 1972, Kim and Takahashi found that $\mathrm{Fe}_{16} \mathrm{~N}_{2}$ has a large saturation magnetization of $28.3 \mathrm{kG}(2.83 \mathrm{~T}) .{ }^{1}$ By using the Mössbauer method, Nakajima and Okamoto then measured the magnetic moment of $\mathrm{Fe}_{16} \mathrm{~N}_{2}$ thin film prepared by the ion-implantation method $\left(2.5 \mu_{\mathrm{B}}\right) .^{2}$ In 1990 Sugita et al. reported that $\mathrm{Fe}_{16} \mathrm{~N}_{2}$ thin films prepared by the molecular beam epitaxy method have a very large saturation magnetization of $32.0 \mathrm{kG}(3.20 \mathrm{~T}) .^{3}$ They reported that the value corresponds to $3.5 \mu_{\mathrm{B}}$ per $\mathrm{Fe}$ atom. ${ }^{3}$ Since then, $\mathrm{Fe}_{16} \mathrm{~N}_{2}$ has attracted much attention as a new magnetic material and many experimental studies have been reported. The saturation magnetization of $\mathrm{Fe}_{16} \mathrm{~N}_{2}$ has been reported to have various values, in the range from 24.0 to $29.3 \mathrm{kG}^{4-11}$ Since then, there have been many discussions regarding whether or not $\mathrm{Fe}_{16} \mathrm{~N}_{2}$ has a large magnetic moment.

In order to resolve this problem, many theoretical band calculations were also performed for $\mathrm{Fe}_{16} \mathrm{~N}_{2}$ in the early 1990s. First, Sakuma calculated the band structure of $\mathrm{Fe}_{16} \mathrm{~N}_{2}$ (Ref. 12) and pointed out that the number of electrons in the minority spin band of each Fe atom obeys Kanamori's rule. ${ }^{13}$ According to Sakuma's results, the average magnetic moment of the Fe atom is $2.39 \mu_{\mathrm{B}}$, which is much smaller than that reported by Sugita et al..$^{3}$ Because of this discrepacy, further electronic band calculations have been performed by several methods (see Table III). The calculated average magnetic moment per Fe atom based on a local density approximation (LDA) is, however, in the range of $2.25-2.50 \mu_{\mathrm{B}}$, which is much smaller than the experimental results. Therefore, it cannot be concluded whether $\mathrm{Fe}_{16} \mathrm{~N}_{2}$ has a large magnetic moment. It should be noted here that the crystallographic data suggested by Jack $^{14}$ were used in these calculations. In the 1950 s, Jack determined the crystal structure of $\mathrm{Fe}_{16} \mathrm{~N}_{2}$ by the $\mathrm{x}$-ray diffraction method with a specimen of tempered $\mathrm{Fe}-\mathrm{N}$ martensite powder. However, only one superstructure reflection line could be observed by the method because of the poor quality of the x-ray film. Thus the structure model was estimated with some ambiguity of the positional parameters.

Recently, Tanaka et al. investigated in detail the crystal structure of $\mathrm{Fe}_{16} \mathrm{~N}_{2}$ precipitated in $\mathrm{Fe}-\mathrm{N}$ martensite, by electron diffraction. ${ }^{15}$ According to the result, some positional parameters in the refined crystal structure differ from those in Jack's model ( $J$ model). A question then arises as to why such a large measured magnetic moment cannot be predicted based on the $J$ model as reported in the previous calculation. It is very important to evaluate theoretically the magnetic moment for the model of Tanaka et al. ( $T$ model). Thus, in this work, the electronic band structures are calculated for the $T$ model, $J$ model, and intermediate structures of both models in which $\mathrm{Fe}(8 h)$ atoms are displaced along the [110] direction.

\section{CRYSTAL STRUCTURE OF $\mathrm{Fe}_{16} \mathrm{~N}_{2}$}

Figure 1 shows the unit cell of $\mathrm{Fe}_{16} \mathrm{~N}_{2}$ in the $T$ model. ${ }^{15}$ Table I lists its crystallographic parameters together with the parameters in the $J$ model. In this table, the $x$ parameter of $\mathrm{Fe}(8 h)$ in the $T$ model is smaller than that in the $J$ model, whereas the $z$ parameter of $\mathrm{Fe}(4 e)$ is almost the same. Figure 2 shows the (101) cross section of the $T$ model. $\mathrm{Fe}(8 h)$ atoms are aligned on the dashed line in the $J$ model. As seen in this figure, $\mathrm{Fe}(8 h)$ atoms shift to the $\mathrm{N}$ atom side from the dashed line and form a zigzag arrangement along the [001] axis. Consequently, $\mathrm{Fe}(8 h)$ atoms are the first-nearest neighbors of the $\mathrm{N}$ atoms in the $T$ model, while $\mathrm{Fe}(4 e)$ atoms are the first-nearest neighbors in the $J$ model. Such changes in atomic configurations are expected to cause some changes in the electronic structure. Thus, in the present study, calculations are performed for several structural models with different values of $\Delta x$ in Table $\mathrm{I}$, where $\Delta x$ is the deviation of $\mathrm{Fe}(8 h)$ from $x=1 / 4$, i.e., $x=1 / 4-\Delta x$. The structures with $\Delta x=0.030$ and $\Delta x=0.000$ correspond to the $T$ model and the $J$ model, respectively, although the parameter $z$ is slightly different from that in the actual $J$ model. Calculations are also performed for intermediate structures where the values of $\Delta x$ are $0.005,0.010,0.015,0.020$, and 0.025 .

\section{CALCULATION METHOD}

Electronic band structures are calculated by a fullpotential linear augmented plane-wave (FLAPW) method. 


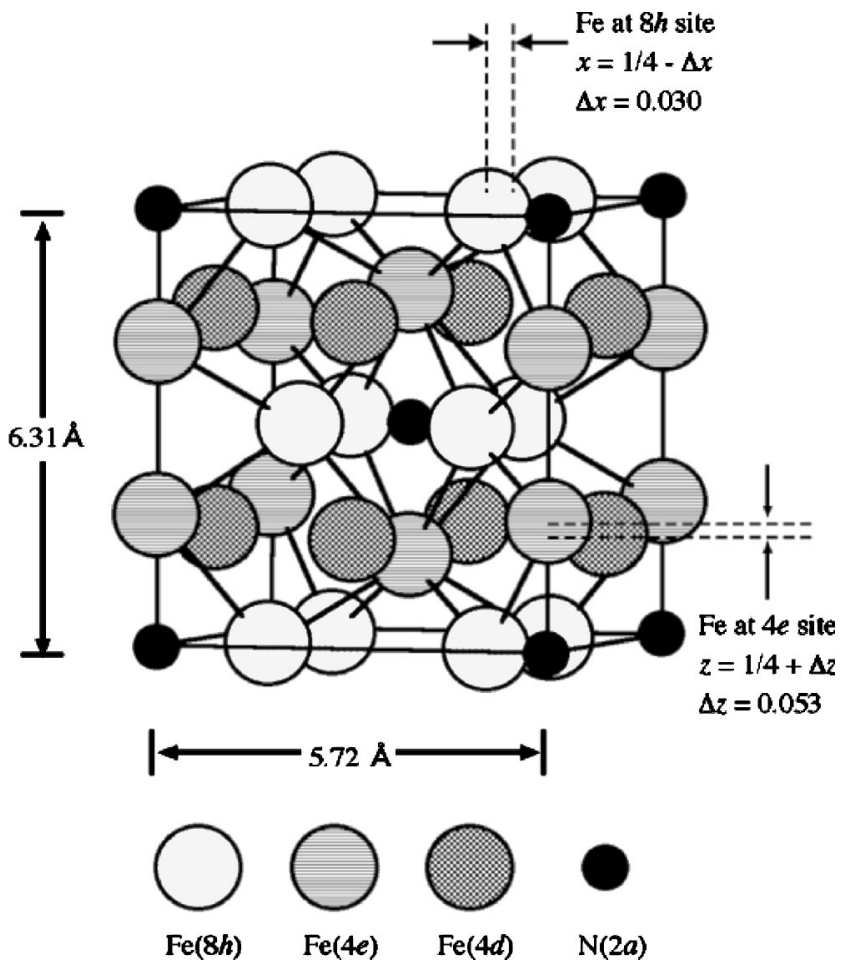

FIG. 1. Crystal structure of $\mathrm{Fe}_{16} \mathrm{~N}_{2}$ refined by Tanaka et al. (Ref. 15).

Because there is strong cobonding between $\mathrm{Fe}$ and $\mathrm{N}$ atoms, particularly in the $\mathrm{Fe}_{16} \mathrm{~N}_{2}$ system, the potential distribution of atoms deviates from a spherical symmetry. Thus the full potential is used in this work. The calculations are carried out self-consistently on the basis of the local spin-density approximation formulated by von Barth and Hedin. ${ }^{16}$ The LAPW functions with the wave vector $\left|\boldsymbol{k}+\boldsymbol{G}_{i}\right| \leqslant \boldsymbol{K}_{\max }=7.2$ $\times(2 \pi / a)$, where $a$ is an $a$-axial lattice constant of $\mathrm{Fe}_{16} \mathrm{~N}_{2}, \boldsymbol{k}$ is a wave vector in the first Brillouin zone, and $\boldsymbol{G}_{i}$ is a reciprocal-lattice vector, are used, leading to about 850 basis functions. Inside the muffin-tin spheres, the angular momentum expansion for the wave function is truncated at $l_{\max }=7$. One-electron energies are obtained at 57 inequivalent $\boldsymbol{k}$ points in the irreducible Brillouin zone. The maximum difference between the magnetic moments for the $\boldsymbol{k}$ points of 57 and 115 is less than $1.0 \times 10^{-3} \mu_{\mathrm{B}}$; thus the number of $\boldsymbol{k}$ points is sufficiently large to ensure the convergence of the

TABLE I. Crystal structure parameters in the $T$ model and the $J$ model.

\begin{tabular}{|c|c|}
\hline & $T$ model \\
\hline \multirow{7}{*}{$\begin{array}{l}\text { Space group } \\
\text { Lattice constant } \\
\text { Atom position }\end{array}$} & $I 4 / \mathrm{mmm}$ \\
\hline & $a=5.72 \AA, c=6.31 \AA \quad a=5.72 \AA, c=6.29 \AA$ \\
\hline & $+(0,0,0)+\left(\frac{1}{2}, \frac{1}{2}, \frac{1}{2}\right)$ \\
\hline & $\mathrm{N}(2 a) \quad 0,0,0$ \\
\hline & $\operatorname{Fe}(8 h) x, x, 0 \quad \bar{x}, x, 0 \quad x, \bar{x}, 0 \quad \bar{x}, \bar{x}, 0$ \\
\hline & $\mathrm{Fe}(4 e) \quad 0,0, z \quad 0,0, \bar{z}$ \\
\hline & $\mathrm{Fe}(4 d) 0, \frac{1}{2}, \frac{1}{4} \frac{1}{2}, 0, \frac{1}{4}$ \\
\hline \multirow{2}{*}{$\begin{array}{l}\text { Positional } \\
\text { parameter }\end{array}$} & $\operatorname{Fe}(8 h)$ with $x=0.220 \quad \operatorname{Fe}(8 h)$ with $x=0.25$ \\
\hline & $\mathrm{Fe}(4 e)$ with $z=0.303$ \\
\hline
\end{tabular}

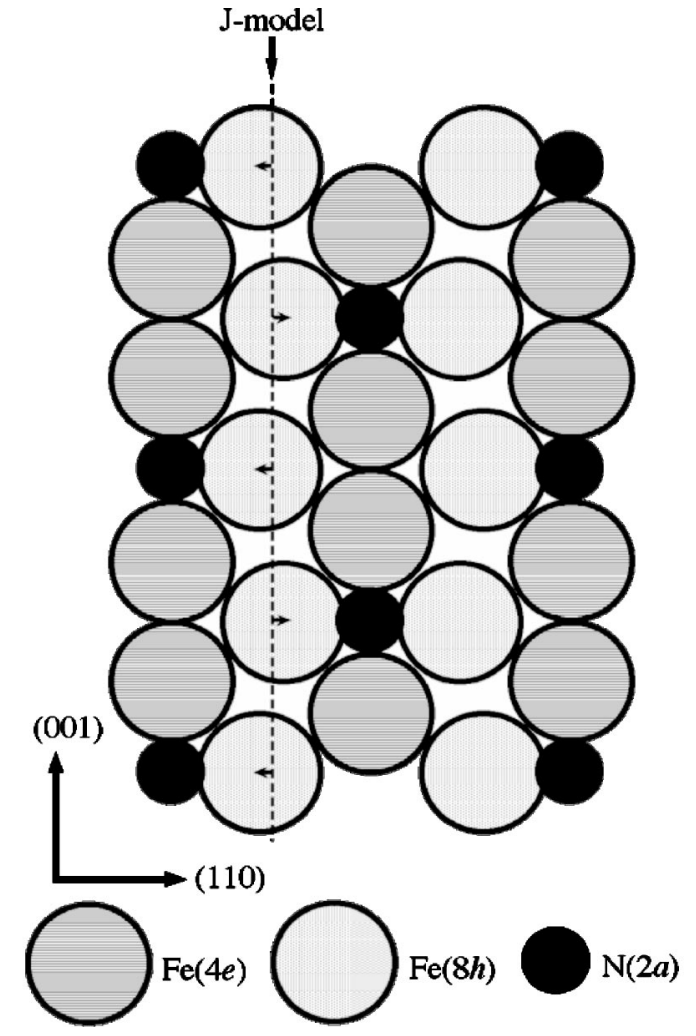

FIG. 2. (101) cross section of $\mathrm{Fe}_{16} \mathrm{~N}_{2}$. $\mathrm{Fe}(8 h)$ atoms shift to the $\mathrm{N}$ atom side from the dashed line and form a zigzag arrangement along the [001] axis.

magnetic moment. The local magnetic moments of $\mathrm{N}$ and $\mathrm{Fe}$ atoms are obtained by integrating the spin density over the muffin-tin spheres with radii of $0.51 \AA$ and $1.13 \AA$, respectively. Because these radii differ from those in the previous studies, ${ }^{17,18}$ the results in this work cannot be compared directly with those in previous ones. Density of states (DOS) is calculated by a tetrahedron method.

\section{RESULTS}

Figure 3 shows the partial DOS of the $p$ band of the $\mathrm{N}$ site and the $d$ bands of three types of $\mathrm{Fe}$ sites in the present FLAPW calculation for $\Delta x=0.030$, which corresponds to the $T$ model. In Fig. 3, sharp peaks appear in the partial DOS of the $p$ states of $\mathrm{N}, \mathrm{Fe}(8 h)$, and $\mathrm{Fe}(4 e)$ at around $0.0 \mathrm{Ry}$. These peaks are considered to be due to the $p$ orbit of $\mathrm{N}$ hybridizing with the states of $\mathrm{Fe}(8 h)$ and $\mathrm{Fe}(4 e)$. The DOS profiles of $\mathrm{Fe}(8 h)$ and $\mathrm{Fe}(4 e)$ are similar to each other and also to that of bcc Fe. In the majority spin bands of $\mathrm{Fe}(8 h)$ and $\mathrm{Fe}(4 e)$, the tail of the main peak extends to the highenergy region above the Fermi level, $E_{\mathrm{F}}$. On the other hand, the partial DOS of $\mathrm{Fe}(4 d)$ is low above $E_{\mathrm{F}}$. This is due to the band narrowing of $\mathrm{Fe}(4 d)$ orbits, because $\mathrm{Fe}(4 d)$ is far from the $\mathrm{N}$ site and is not influenced by other $\mathrm{Fe}$ or $\mathrm{N}$ atoms. $\mathrm{Fe}(4 d)$ has the $3 d$ hole in the minority spin states immediately above $E_{\mathrm{F}}$. Thus, as listed in Table II, the number of $\mathrm{Fe}(4 d)$ electrons is larger in the majority spin band and smaller than those of other Fe sites in the minority spin band. Consequently, the magnetic moment of $\mathrm{Fe}(4 d)$ is $2.7 \mu_{\mathrm{B}}$, larger than those of other sites of Fe, as listed in Table III, which also gives several other calculated results for compari- 

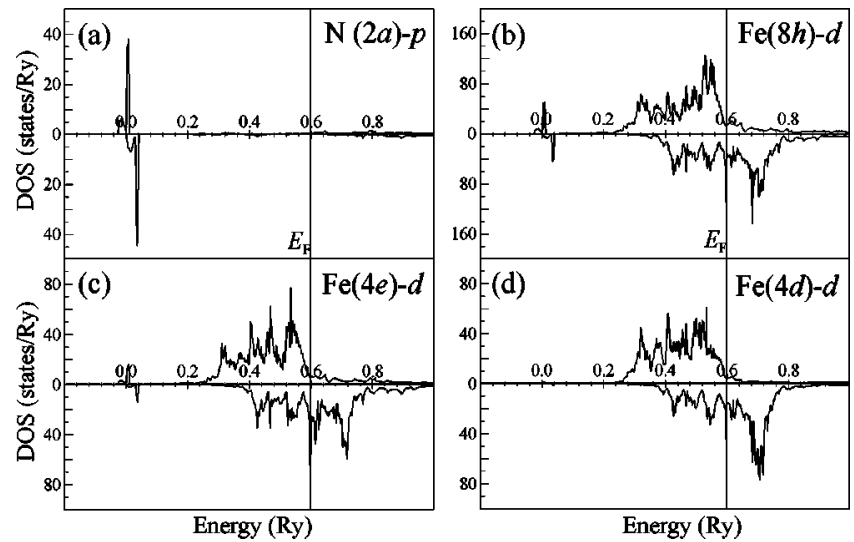

FIG. 3. Partial DOS of $\mathrm{Fe}_{16} \mathrm{~N}_{2}$ for the $T$ model. (a) $p$ band of $\mathrm{N}(2 a)$, (b) $d$ band of $\mathrm{Fe}(8 h)$, (c) $d$ band of $\mathrm{Fe}(4 e)$, and (d) $d$ band of $\mathrm{Fe}(4 d)$. Sharp peaks appear in the partial DOS of the $p$ state of $\mathrm{N}, \mathrm{Fe}(8 h)$, and $\mathrm{Fe}(4 e)$ at around $0.0 \mathrm{Ry}$. These peaks are considered to be due to the $\mathrm{N} p$ orbit hybridizing with $\mathrm{Fe}(8 h)$ and $\mathrm{Fe}(4 e)$ states. The DOS profiles of $\mathrm{Fe}(8 h)$ and $\mathrm{Fe}(4 e)$ are similar to each other and also to that of bcc-Fe.

son. The calculated value of the average moment per $\mathrm{Fe}$ atom in $\mathrm{Fe}_{16} \mathrm{~N}_{2}$ is not as large as that of the experimental results reported by Sugita $e t$ al. ${ }^{3}$

Figure 4 shows the partial DOS of $\mathrm{N}$ and three types of $\mathrm{Fe}$ sites in the present FLAPW calculation for $\Delta x=0.000$, which corresponds to the $J$ model. The partial DOS of each $\mathrm{Fe}$ site in Fig. 4 is very similar to that of bcc Fe. The profile of bcc Fe has two large peaks corresponding to the states of $d \gamma$ and $d \varepsilon$, around 0.8 and 1.0 Ry in the up spin and around 0.9 Ry and $1.2 \mathrm{Ry}$ in the down spin, respectively. ${ }^{19}$ On the other hand, the DOS profile of fcc Fe has only one broad peak. ${ }^{20}$ The DOS profile in the $T$ model (Fig. 3) deviates from that in the $J$ model (Fig. 4), and we cannot distinguish between the two main peaks due to the $d \gamma$ and $d \varepsilon$ states. Where $\Delta x=0.030$, the two peaks cannot be distinguished in the DOS profiles of $\mathrm{Fe}(4 e)$ and $\mathrm{Fe}(8 h)$. This is because the ligand field of $\mathrm{Fe}$ atoms deviates from that which is similar to the bcc Fe field to an intermixed field of bcc Fe and fcc $\mathrm{Fe}$. The local magnetic moment and number of electrons calculated by the FLAPW method against the parameter $\Delta x$ are shown in Figs. 5(a) and 5(b), respectively. Where $\Delta x$ is 0.000 , the moment of $\mathrm{Fe}$ sites increases in the order of $\mathrm{Fe}(4 e), \mathrm{Fe}(8 h)$, and $\mathrm{Fe}(4 d)$. On the other hand, charge increases in the order of $\mathrm{Fe}(4 d), \mathrm{Fe}(8 h)$, and $\mathrm{Fe}(4 e)$. This figure shows that the number of minority spins is mainly changed in both $\mathrm{Fe}(4 e)$ and $\mathrm{Fe}(8 h)$ against $\Delta x$. The moment and the number of electrons of $\mathrm{Fe}(8 h)$ and $\mathrm{Fe}(4 e)$ cross over where $\Delta x$ is around 0.015 . This may be related to

TABLE II. Local charge of $\mathrm{Fe}_{16} \mathrm{~N}_{2}$ in this work.

\begin{tabular}{llcccc}
\hline \hline & & \multicolumn{4}{c}{ Number of electrons } \\
& & $\mathrm{N}(2 a)$ & $\mathrm{Fe}(8 h)$ & $\mathrm{Fe}(4 e)$ & $\mathrm{Fe}(4 d)$ \\
\hline$\Delta x=0.000$ & majority & 1.68 & 13.37 & 13.27 & 13.57 \\
$(J$ model $)$ & minority & 1.70 & 11.09 & 11.29 & 10.79 \\
$\Delta x=0.030$ & majority & 1.70 & 13.27 & 13.33 & 13.54 \\
$(T$ model $)$ & minority & 1.74 & 11.32 & 11.15 & 10.79 \\
\hline \hline
\end{tabular}

TABLE III. Local magnetic moment of $\mathrm{Fe}_{16} \mathrm{~N}_{2}$ in this work and previous studies.

\begin{tabular}{|c|c|c|c|c|c|}
\hline & $\mathrm{N}(2 a)$ & $\mathrm{Fe}(8 h)$ & $\mathrm{Fe}(4 e)$ & $\operatorname{Fe}(4 d)$ & $\begin{array}{l}\mathrm{Ave} / \mathrm{Fe} \\
\text { atom }\end{array}$ \\
\hline LMTO (Ref. 12) & -0.07 & 2.25 & 2.27 & 2.83 & 2.39 \\
\hline LMTO (Ref. 21) & & 2.41 & 1.96 & 2.91 & 2.42 \\
\hline LMTO (Ref. 22) & 0.06 & 2.50 & 2.13 & 2.85 & 2.50 \\
\hline ASW (Ref. 23) & -0.01 & 2.37 & 2.30 & 2.74 & 2.42 \\
\hline FLAPW (Ref. 17) & -0.03 & 2.33 & 2.04 & 2.82 & 2.37 \\
\hline DV-X $\alpha$ (Ref. 24) & & 2.26 & 1.71 & 2.87 & 2.27 \\
\hline LMTO (Ref. 8) & & 2.39 & 2.21 & 2.81 & 2.45 \\
\hline APW (Ref. 25) & -0.043 & 2.295 & 2.000 & 2.757 & 2.403 \\
\hline $\begin{array}{l}\mathrm{LMTO}(\mathrm{LDA}+U) \\
\quad(\text { Ref. 26) }\end{array}$ & -0.01 & 2.75 & 2.36 & 3.53 & 2.85 \\
\hline OLCAO (Ref. 27) & -0.06 & 2.42 & 2.06 & 2.90 & 2.44 \\
\hline $\begin{array}{l}\text { Exp. Mössbauer } \\
\text { (Ref. 2) }\end{array}$ & & 2.5 & 1.3 & 3.8 & 2.5 \\
\hline This work ( $J$ model $)$ & -0.02 & 2.28 & 1.97 & 2.78 & 2.32 \\
\hline This work ( $T$ model) & -0.03 & 1.95 & 2.17 & 2.74 & 2.20 \\
\hline
\end{tabular}

the fact that the $\mathrm{Fe}(8 h)$ site becomes the first-nearest neighbor from the second-nearest neighbor of the $\mathrm{N}$ site where $\Delta x$ is larger than 0.014. The moment of $\mathrm{Fe}(4 d)$ is always larger and the number of electrons of $\mathrm{Fe}(4 d)$ is always smaller than that of other Fe sites. The moment and number of electrons of $\mathrm{Fe}(4 d)$ are almost constant and independent of the displacement of $\mathrm{Fe}(8 h), \Delta x$. Figure 6 shows the calculated total energy, $E_{\text {tot }}$, of the $\mathrm{Fe}_{16} \mathrm{~N}_{2}$ lattice against the displacement of $\mathrm{Fe}(8 h), \Delta x$. The total energy can be fitted by a parabolic function, which has a minimum at $\Delta x=0.008$. The difference between $\Delta x$ 's for the numbers of plane waves of 650 and 850 is $\sim 10^{-4}$, which is sufficiently small to obtain the same optimized atomic position. This shows that $\mathrm{Fe}$ atoms at $8 h$ site clearly deviate from the high symmetric position in the $J$ model $(\Delta x=0)$ to the low one.

\section{DISCUSSION}

First, we attempt to discuss the results for the $T$ model. As described above, the local magnetic moment of each $\mathrm{Fe}$ in
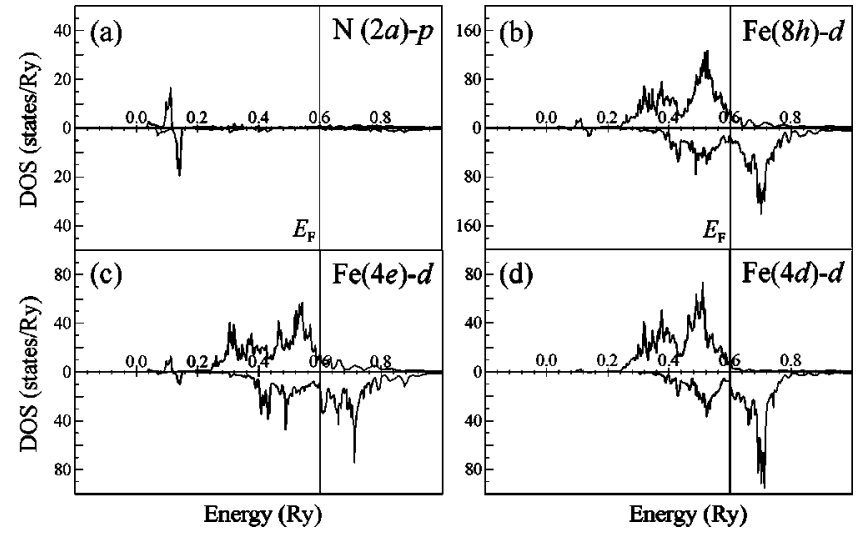

FIG. 4. Partial DOS of $\mathrm{Fe}_{16} \mathrm{~N}_{2}$ for the $J$ model. (a) $p$ band of $\mathrm{N}(2 a),(\mathrm{b}) d$ band of $\mathrm{Fe}(8 h)$, (c) $d$ band of $\mathrm{Fe}(4 e)$, and (d) $d$ band of $\mathrm{Fe}(4 d)$. 

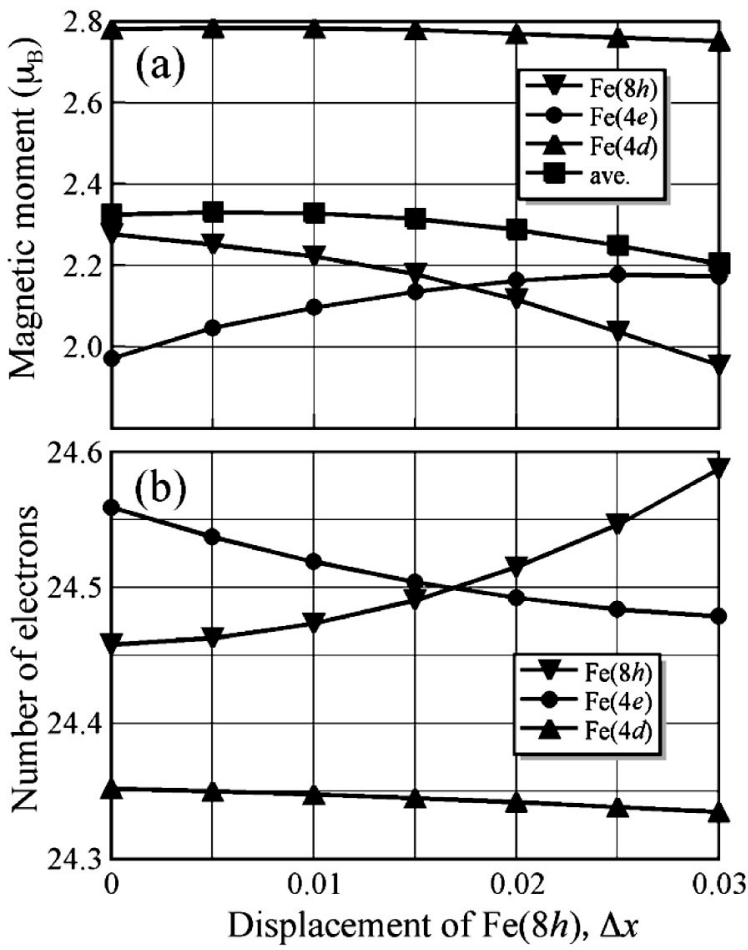

FIG. 5. (a) Local magnetic moment and (b) local number of electrons against the displacement of $\mathrm{Fe}$ atoms at the $8 h$ site, $\Delta x$.

$\mathrm{Fe}_{16} \mathrm{~N}_{2}$ is considerably different depending on the site. It should be noted that the $\mathrm{Fe}$ site most distant from the $\mathrm{N}$ site, $\mathrm{Fe}(4 d)$, has the largest $3 d$ unoccupied states in the down spin, while the majority spin states of the site are mostly occupied. In fact, the local magnetic moments of this site are the largest, as shown in Table III. The number of $\mathrm{Fe}(4 d)$ electrons is found to be less (about 0.15 to 0.25 ) than that at other sites, as shown in Fig. 5(b). Therefore, it can be considered that the large moments of $\mathrm{Fe}(4 d)$ are mainly due to the shortage of minority spin electrons rather than to the receipt of majority spin electrons. The variations of these charge distributions on each $\mathrm{Fe}$ site can be regarded as an effect of the $\mathrm{N}$ atom. Thus the present result that the moments of $\mathrm{Fe}(8 h)$ and $\mathrm{Fe}(4 e)$ in $\mathrm{Fe}_{16} \mathrm{~N}_{2}$ are smaller than that of $\mathrm{Fe}(4 d)$ is due to the fact that their distances from the $\mathrm{N}$ atom are much shorter than for $\mathrm{Fe}(4 d)$.

Next we discuss the results of comparing the $T$ model with the $J$ model. We focus on the DOS profiles of $\mathrm{Fe}(4 e)$ and $\mathrm{Fe}(8 h) . \mathrm{Fe}(4 d)$ is the third-nearest neighbor of an $\mathrm{N}$ atom in both models. Thus some features of $\mathrm{Fe}(4 d)$ are the same in the $T$ model and the $J$ model. The weak hybridization with an $\mathrm{N}$ atom is a common feature in both Fig. 3(d)

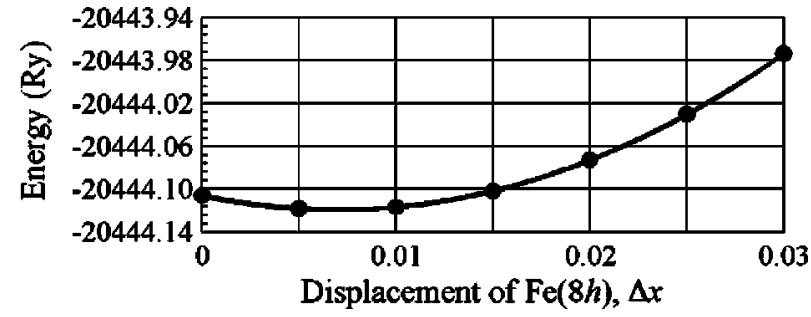

FIG. 6. Total energy of $\mathrm{Fe}_{16} \mathrm{~N}_{2}$ against the displacement of $\mathrm{Fe}$ atoms at the $8 h$ site, $\Delta x$. and Fig. 4(d) because $\mathrm{N} p$ states do not appear in the DOS profile of $\mathrm{Fe}(4 d)$. Moreover, the DOS profile of $\mathrm{Fe}(4 d)$ decreases steeply at the top of the $d$ band although those of $\mathrm{Fe}(8 h)$ and $\mathrm{Fe}(4 e)$ have tails to the high-energy side. Ishida et al. ${ }^{21}$ have shown that this tail is due to the effect of $\mathrm{N}$ atoms, on the basis of band calculations for $\mathrm{Fe}_{16} \mathrm{~N}_{0}$, which has $\mathrm{Fe}$ atoms at the same positions as in $\mathrm{Fe}_{16} \mathrm{~N}_{2}$ but no $\mathrm{N}$ atom at the $\mathrm{N}$ site. According to their calculations, there are no tails at the top of the $d$ electron band, not only in partial DOS for $\mathrm{Fe}(4 d)$ but also in partial DOS for $\mathrm{Fe}(8 h)$ and $\mathrm{Fe}(4 e)$. They concluded that $\mathrm{N}$ atoms cause band broadening, which signifies that the distance from an $\mathrm{N}$ atom is decreasing. This rule should also be satisfied in the case of the $T$ model. Thus the tail of DOS for the $T$ model, as well as that for the $J$ model, can be attributed to the effects of $\mathrm{N}$ atoms. In the present work, the number of electrons in the minority spin band of each $\mathrm{Fe}$ atom obeys Kanamori's rule ${ }^{13}$ described above. As shown in Fig. 3, the occupied states of the minority spin band increase with increasing atomic distance from an $\mathrm{N}$ atom. Accordingly, the presence of $\mathrm{N}$ atoms reduces the magnetic moment of $\mathrm{Fe}_{16} \mathrm{~N}_{2}$.

The magnetic moment obtained for the $T$ model in the present work is not as large as that obtained in the previous experiment. We attempt to explain why there is so large a difference between the experimental and the calculated results. One of the reasons is a limitation of LDA, which is the approximation that electrons distribute as a homogenous gas under any circumstance. Hence an error occurs when localized electrons are present in the system. Therefore, the magnetic moment may be underestimated. Another reason concerns the experimental side. If the practical magnetic moment of $\mathrm{Fe}_{16} \mathrm{~N}_{2}$ is as small as the calculated result, another Fe-N material that has a large magnetic moment should be present in the Fe-N specimen. Previously, Mitsuoka et al. reported that $\mathrm{Fe}-11.1$ at. $\% \mathrm{~N}$ martensite, the structure of which contains $\mathrm{N}$ atoms existing randomly at octahedral interstices of bct-Fe, might theoretically have a large magnetic moment of $2.61 \mu_{\mathrm{B}} \cdot{ }^{28}$ This value was estimated by extrapolating the magnetic moment of $\mathrm{Fe}-\mathrm{N}$ martensite. According to the results of recently performed high-resolution electron microscopy observations, ${ }^{29,30} \mathrm{Fe}-\mathrm{N}$ martensite containing slightly less than 11 at. \% nitrogen (high-nitrogen martensite) is present around the matrix and $\mathrm{Fe}_{16} \mathrm{~N}_{2}$. If there is a highnitrogen martensite in which nitrogen is slightly decreased from 11 at. \%, the phase also changes its electronic structure from that of the $\mathrm{Fe}_{16} \mathrm{~N}_{2}$ phase. Thus the presence of the high-nitrogen martensite is one of the reasons for the large magnetic moment of the $\mathrm{Fe}-\mathrm{N}$ system.

It is an important result that the total energy indicates that $\Delta x=0.000$ is not a stable position of $\mathrm{Fe}(8 h)$. This shows that the symmetry of $\mathrm{Fe}(8 h)$ changes. Because the atomic symmetry determines the base functions, it is clear that different properties are derived by calculation between $\Delta x$ $=0.000$ and other $\Delta x$ 's. Because most previous works were performed with $\Delta x=0.000$, we could only determine the tendency of the magnetic properties of $\mathrm{Fe}_{16} \mathrm{~N}_{2}$. Hence the result gives us a new way to understand the magnetism of $\mathrm{Fe}_{16} \mathrm{~N}_{2}$. The reason why the stable position does not coincide with the experimental value, $\Delta x=0.030$, may be that the LDA was used for the density of electrons. In the case where electrons are localized by the distance between $\mathrm{N}$ and Fe being 
very narrow, the total energy found by this calculation method tends to be large. The lattice parameter is fixed for the experimental value in this study. Because the distance between $\mathrm{Fe}(8 h)$ and $\mathrm{N}$ decreases when $\Delta x$ increases, the total energy is estimated to be larger than the essential one. Considering that the total energy does not become minimum at the experimental lattice parameter $(\Delta x=0.030)$, we must perform further studies using a more precise calculation method than that used in this study, such as LDA $+U$.

\section{SUMMARY}

The partial DOS of $\mathrm{Fe}$ in $\mathrm{Fe}_{16} \mathrm{~N}_{2}$ shows that the ligand field of $\mathrm{Fe}$ atoms deviates from a field that is similar to bcc-Fe to an intermixed field of bcc-Fe and fcc-Fe by displacement of $\mathrm{Fe}(8 h)$ from $\Delta x=0.000$ to 0.030 . The magnetic moments of $\mathrm{Fe}(8 h)$ and $\mathrm{Fe}(4 e)$ depend strongly on the displacement of $\mathrm{Fe}(8 h), \Delta x$. The total energy indicates that $\Delta x=0.000$ is not a stable position of $\mathrm{Fe}(8 h)$. The magnetic moment of $\mathrm{Fe}(4 d)$ does not strongly depend on $\Delta x$ because
$\mathrm{Fe}(4 d)$ is distant from $\mathrm{N}$ atoms. The average magnetic moment per $\mathrm{Fe}$ atom of $\mathrm{Fe}_{16} \mathrm{~N}_{2}$ becomes maximum at $\Delta x$ $=0.005$. There are some reasons for the discrepancy between the experimental and the theoretical results for the magnetic moment. One of the reasons is the presence of another phase or material that has a large magnetic moment; this must be considered in the explanation of the large magnetic moment of the Fe-N system.

\section{ACKNOWLEDGMENTS}

The computation in this work was performed using the facilities of the Supercomputer Center, Institute for Solid State Physics, University of Tokyo. The authors thank Professor Yanase of the ISIR, Osaka University and Dr. Sakuma of Hitachi Metals Ltd., for their useful discussions. One of the authors (H.T.) acknowledges the Japan Society for the Promotion of Science. H.T. also thanks T. Ohkubo and Dr. K. Betsuyaku of ISIR, Osaka University for their support.
*Corresponding author. Present address: Surface and Interface Laboratory, RIKEN (The Institute of Physical and Chemical Research), Hirosawa 2-1, Wako, Saitama 351-0198, Japan. Email address: tanaka@postman.riken.go.jp

†Present address: Kochi University of Technology, Miyanokuchi 185, Tosa-Yamada, Kochi 782-8502, Japan.

Present address: College of Science and Engineering, Iwaki Meisei University, Iino 5-5-1, Chuo-dai, Iwaki, Fukushima 9708551, Japan.

${ }^{1}$ T.K. Kim and M. Takahashi, Appl. Phys. Lett. 20, 492 (1972).

${ }^{2}$ K. Nakajima and S. Okamoto, J. Appl. Phys. 65, 4357 (1989).

${ }^{3}$ Y. Sugita, K. Mitsuoka, M. Komuro, H. Hoshiya, Y. Kozono, and M. Hanazono, J. Appl. Phys. 70, 5977 (1991).

${ }^{4}$ C. Gao, W. Doyle, and M. Shamsuzzoha, J. Appl. Phys. 73, 6579 (1993).

${ }^{5}$ C. Oritz, G. Dumpich, and A.H. Morrish, Appl. Phys. Lett. 65, 2737 (1994).

${ }^{6}$ X. Bao, R.M. Metzger, and M. Carbucicchio, J. Appl. Phys. 75, 5870 (1994).

${ }^{7}$ W.E. Wallace and Q. Huang, J. Appl. Phys. 76, 6648 (1994).

${ }^{8}$ J.M. Coey, K. O'Donnell, Q. Qinian, E. Touchais, and K.H. Jack, J. Phys.: Condens. Matter 6, L23 (1994).

${ }^{9}$ S. Okamoto, O. Kitakami, and Y. Shimada, J. Appl. Phys. 79, 5250 (1996).

${ }^{10}$ M. Takahashi, H. Shoji, H. Takahashi, H. Nashi, T. Wakiyama, M. Doi, and M. Matsui, J. Appl. Phys. 76, 6642 (1994).

${ }^{11}$ M.A. Brewer, K.M. Krishnan, and C. Ortiz, J. Appl. Phys. 79, 5321 (1996).

${ }^{12}$ A. Sakuma, J. Magn. Magn. Mater. 102, 127 (1991).

${ }^{13}$ J. Kanamori, Proceedings of the 10th International Workshop on Rare-Earth Magnets and Their Applications, Kyoto, 1989 (The Society of Non-Traditional Technology, Tokyo, 1989), Vol. 1, p. 1.; the magnetic moment of the next-nearest neighbor of $\mathrm{B}, \mathrm{C}$, and $\mathrm{N}$ is indirectly enhanced by the increase in energy levels of the minority spin states through a direct lowering of the minority spin states of the first-nearest-neighbor $\mathrm{Fe}$ atoms.

${ }^{14}$ K.H. Jack, Proc. R. Soc. London, Ser. A 208, 200 (1951).

${ }^{15}$ H. Tanaka, S. Nagakura, Y. Nakamura, and Y. Hirotsu, Acta Mater. 45, 1401 (1997).

${ }^{16}$ U. von Barth and L. Hedin, J. Phys. C 5, 1629 (1972).

${ }^{17}$ R. Coehoorn, G.H.O. Daalderop, and H.J.F. Jansen, Phys. Rev. B 48, 3830 (1993).

${ }^{18}$ H. Tanaka, H. Harima, T. Yamamoto, H. Katayama-Yoshida, Y. Nakata, and Y. Hirotsu, J. Magn. Magn. Mater. 177-181, 1468 (1998).

${ }^{19}$ T. Sasaki, A.M. Rappe, and S.G. Louie, Phys. Rev. B 52, 12760 (1995).

${ }^{20}$ For example, Calculated Electronic Properties of Ordered Alloys: A Handbook: The Elements and Their 3d-3d and $4 d-4 d$ Alloys, edited by V.L. Moruzzi and C.B. Sommers (World Scientific, Singapore, 1995).

${ }^{21}$ S. Ishida, K. Kitawatase, S. Fujii, and S. Asano, J. Phys.: Condens. Matter 4, 765 (1992).

${ }^{22}$ B.I. Min, Phys. Rev. B 46, 8232 (1992).

${ }^{23}$ S. Mater, Z. Phys. B: Condens. Matter 87, 91 (1992).

${ }^{24}$ K. Miura, S. Imanaga, and Y. Hayafuji, J. Phys.: Condens. Matter 5, 9393 (1993).

${ }^{25}$ H. Sawada, A. Nogami, T. Matsumiya, and T. Oguchi, Phys. Rev. B 50, 10004 (1994).

${ }^{26}$ W.Y. Lai, Q.Q. Zheng, and W.Y. Hu, J. Phys.: Condens. Matter 6, L259 (1994).

${ }^{27}$ M.Z. Huang and W.Y. Ching, Phys. Rev. B 51, 3222 (1995).

${ }^{28}$ K. Mitsuoka, H. Miyajima, H. Ino, and S. Chikazumi, J. Phys. Soc. Jpn. 53, 2381 (1984).

${ }^{29}$ H. Tanaka and Y. Hirotsu, J. Magn. Soc. Jpn. 23, 858 (1999).

${ }^{30} \mathrm{H}$. Tanaka and Y. Hirotsu, in 14th International Conference on Electron Microscopy, Cancun, Mexico, 1998, edited by H. A. C. Benavides and M. J. Yacaman (Institute of Physics, Bristol, 1998), p. 553. 